\title{
HIV-Associated Tuberculosis: Diagnostic and Treatment Challenges
}

\author{
Wafaa M. EI-Sadr, M.D., M.P.H. ${ }^{1,2}$ and Simon J. Tsiouris, M.D., M.P.H. ${ }^{3,4}$
}

Tuberculosis (TB) and the human immunodeficiency virus (HIV) are, individually, two of the world's greatest ongoing public health threats. In combination, the two diseases can be even more devastating. HIV significantly increases an individual's chances of reactivation of latent TB infection and progression to active TB disease. HIV's associated immunosuppression makes it more difficult to diagnose active TB due to a higher likelihood of atypical and extrapulmonary presentation and poorer performance of standard diagnostic tools. TB is the major cause of death in individuals infected with HIV, and the combination of both illnesses creates unique treatment challenges for providers due to interactions between antituberculous and antiretroviral medications, overlapping drug toxicities, and the immune reconstitution inflammatory syndrome. Magnifying these challenges even further is the fact that much of the burden of TB/HIV coinfection exists in some of the world's most resource-limited settings. Concerted efforts are needed to identify rapid and accurate diagnostic tools for active TB disease and latent TB infection (LTBI) that are practical and inexpensive and that perform well in individuals with HIV infection. Also needed are effective and feasible strategies to optimize management of both conditions in the coinfected patient.

KEYWORDS: Tuberculosis, human immunodeficiency virus, diagnosis, treatment

Mycobacterium tuberculosis (MTB) and the human immunodeficiency virus (HIV) are pathogens that, individually, are responsible for two of humankind's greatest ongoing health calamities: active tuberculosis (TB) disease, responsible for 1.7 million deaths worldwide in 2006, ${ }^{1}$ and HIV-related diseases, including the acquired immunodeficiency syndrome (AIDS), responsible for 2.1 million deaths worldwide in $2007 .^{2}$ In combination, the two diseases are ever more devastating. In 2006, 700,000 of the 9.2 million new cases of active TB and 200,000 of the 1.7 million deaths due to TB were in individuals also infected with HIV. ${ }^{1}$
HIV dramatically increases an individual's chances of reactivation of latent TB infection (LTBI) and progression to active TB disease many-fold higher than any other risk factor, ${ }^{3-5}$ whereas its associated immunosuppression makes it more difficult to diagnose TB due to a higher likelihood of atypical presentation. TB is firmly entrenched as the major cause of death in individuals infected with HIV and creates challenges for providers when both diseases need to be treated simultaneously due to interactions between some antituberculous medications and certain antiretroviral drugs, overlapping drug toxicities, and the common occurrence
${ }^{1}$ International Center for AIDS Care and Treatment Programs, Mailman School of Public Health, Columbia University, New York, New York; ${ }^{2}$ Division of Infectious Diseases, Harlem Hospital Center, New York, New York; ${ }^{3}$ Division of Infectious Diseases, College of Physicians and Surgeons, Columbia University, New York, New York; ${ }^{4}$ Department of Epidemiology, Mailman School of Public Health, Columbia University, New York, New York.

Address for correspondence and reprint requests: Simon J. Tsiouris,
M.D., M.P.H., Division of Infectious Diseases, College of Physicians and Surgeons, Columbia University, 622 West 168th St., PH 8 876W, New York, NY 10032 (e-mail: st326@columbia.edu).

Tuberculosis; Guest Editor, Neil W. Schluger, M.D.

Semin Respir Crit Care Med 2008;29:525-531. Copyright (C) 2008 by Thieme Medical Publishers, Inc., 333 Seventh Avenue, New York, NY 10001, USA. Tel: +1(212) 584-4662.

DOI 10.1055/s-0028-1085703. ISSN 1069-3424. 
of immune reconstitution inflammatory syndromes. ${ }^{6}$ With the rise of multidrug resistant (MDR) TB worldwide $^{7}$ and extensively drug resistant (XDR) TB in people infected with $\mathrm{HIV}{ }^{8}$ these diagnostic and treatment challenges are steadily multiplying. From a patient's perspective, having both TB and HIV is particularly difficult. Both conditions are associated with stigma and discrimination, and adherence with treatment for both conditions is particularly challenging.

Complicating matters even further is the fact that much of the burden of TB/HIV coinfection exists in some of the world's most resource-limited settings, with the brunt of that burden being borne by sub-Saharan Africa where some countries report an HIV prevalence of greater than $50 \%$ among new cases of TB. ${ }^{1}$ In these resource-limited settings, patients and providers often have little to no access to many of the standard diagnostic and therapeutic options available to overcome some of these challenges, and there is often a paucity of infection control measures, all of which contributes to the ongoing spread of TB.

\section{ACTIVE TUBERCULOSIS}

\section{Diagnostic Challenges}

The presence of HIV infection complicates the clinical presentation of active TB through its effect on the immune system resulting in changes in the presentation of active TB disease, which can make diagnosis of active TB more difficult and delay diagnosis. HIV infection can lead to paucibacillary disease ${ }^{9}$ in patients with active pulmonary $\mathrm{TB}$, which decreases the sensitivity of the acid-fast sputum smear test, ${ }^{10}$ the most widely used and available TB diagnostic method, especially in resourcelimited settings. Patients with HIV, especially advanced HIV disease, and active pulmonary TB are less likely to have "typical" chest radiograph findings such as cavitary lesions and more likely to have chest radiographs demonstrating lymphadenopathy and/or atypical infiltrates. $^{11,12}$ Patients with HIV and active TB are also more likely to have extrapulmonary $\mathrm{TB}$, especially those with lower CD4 $\mathrm{T}$ cell counts. ${ }^{13}$

As a consequence of high rates of sputum smear negativity, atypical chest radiograph findings, and the increased incidence of extra-pulmonary TB, the diagnosis of TB may be missed or delayed in HIV-infected individuals. Smear-negative TB and delayed diagnosis of TB in individuals with HIV have both been associated with increased mortality. ${ }^{14-16}$ Finally, HIV infection has been associated with a high prevalence of subclinical TB in settings of high TB burden, ${ }^{17}$ further complicating diagnosis and leading to the need for effective means of screening for active TB in these populations, especially prior to initiation of treatment for LTBI. ${ }^{18,19} \mathrm{It}$ is therefore imperative to develop new rapid and accurate means for the diagnosis of active TB in HIV-infected patients with subclinical, smear-negative pulmonary and/or extrapulmonary TB.

In resource-limited areas, the tuberculin skin test (TST) is sometimes used to aid in the diagnosis of active TB. Beyond its lack of specificity for this type of application and its decreased sensitivity in individuals with HIV infection, in settings of high TB prevalence and/or high rates of bacille Calmette-Guérin (BCG) vaccination the TST has been shown to have particularly limited utility as a diagnostic aid for active TB.,20 Researchers have looked at improving the speed and accuracy of active TB diagnosis through the use of other types of immunologic assays aimed at detecting TB-specific antibodies ${ }^{21,22}$ and antigens. ${ }^{23}$ Thus far, these strategies, much like the TST, have not yielded results with acceptable sensitivity or specificity.

Breen et al examined improving the rapidity of diagnosing smear-negative tuberculosis by performing rapid immunoassays for MTB purified protein derivative (PPD)-specific CD4 lymphocytes on the induced sputa of traditional sputum-smear-negative or sputum-nonproducing TB suspects. Sixteen of their 42 subjects were HIV infected. Sensitivity and specificity of the immunoassay versus final diagnosis of active TB were $89 \%$ and $80 \%$, respectively. ${ }^{24}$ In combination with a single sputum smear for acid-fast bacilli (AFB) the sensitivity increased to $93 \%$. Their hopeful results were unaffected by the HIV status of the individual.

Commercially available MTB nucleic acid amplification tests (NAATs) have decreased the time to definitive diagnosis of AFB smear positive pulmonary $\mathrm{TB}$ due to their short turnaround time and high sensitivity and specificity. ${ }^{25}$ Resource-limited settings, however, generally lack the laboratory infrastructure necessary to reliably perform NAATs for MTB. In addition, the costs associated with NAATs are prohibitive in resource-limited settings. For these tests to become more widely available in resourcelimited settings, the technology required to perform them must become simpler and their costs must decrease substantially.

The challenges associated with implementing NAAT technology in resource limited settings have not deterred investigators in those settings from examining possible uses of such technologies. In a study conducted in Tanzania on $120 \mathrm{HIV}$-infected TB suspects (28 of whom were subsequently proven to have active TB by culture confirmation), Kibiki et al examined the use of a commercially available whole blood serologic assay for diagnosis of active TB as well as the use of an investigator-developed real-time polymerase chain reaction (PCR) for MTB DNA on bronchoalveolar lavage fluid. The serologic assay fared very poorly in this $\mathrm{HIV}$-infected group of patients, but the PCR assay had a sensitivity of $85.7 \%$ and a specificity of $90.9 \%{ }^{26}$ 
Though performed on small samples of patients and a long way from commercial development and clinical application, especially in settings with limited resources, studies like the two summarized above highlight the inventiveness that will be required to tackle the challenges HIV poses in the diagnosis of TB and lend hope to the possibility of advances in the near future in the rapid and accurate diagnosis of TB in patients with HIV. In this issue of Seminars in Respiratory and Critical Care Medicine, Drs. Pai and O'Brien (article 9) review new diagnostics for active TB.

\section{Treatment Challenges}

The choices, challenges, complications, and future of the treatment of HIV-associated active TB have been recently reviewed. ${ }^{6,27,28}$ In this issue of Seminars, Dr. Nuermberger (article 7) discusses the use of animal models to develop new treatments for $\mathrm{TB}$ and Dr. Ginsberg (article 8) reviews emerging drugs for the treatment of active TB. Treatment of TB and HIV disease in patients with both conditions presents multiple challenges, including the correct timing of initiation of antiretroviral (ARV) therapy during TB treatment, the correct choice of drug combinations to limit interactions, and close patient monitoring to watch for drug toxicities (which often overlap) and other consequences, such as immune reconstitution inflammatory syndrome (IRIS).

It is recommended that antituberculous treatment be promptly initiated upon diagnosis of TB in an effort to decrease mortality and infectiousness. ${ }^{29,30}$ Additionally, emphasis has been placed on starting ARV therapy, when indicated, during TB treatment due to the high morbidity and mortality in patients with HIV coinfection even when receiving appropriate antituberculous treatment. ${ }^{31}$ The World Health Organization recommends that the timing of initiation of ARV therapy in these patients be based on the degree of immunosuppression as defined by CD4 cell count. ${ }^{32}$ Although not an unreasonable approach, further research is necessary to better define the optimal time for initiation of ARV therapy during TB treatment.

Several factors may complicate concurrent ARV therapy, including additive toxicities of medications, drug interactions, risk of immune reconstitution events, and difficulty in medication adherence. ${ }^{33}$ Despite these challenges, use of ARV therapy for those who qualify during TB treatment is important because it improves outcomes and decreases mortality. ${ }^{34}$ The choices of ARVs that can be used safely during TB treatment are severely limited by the necessary use of rifampin (or other rifamycins) in most regimens for the treatment of rifampin-susceptible TB due to rifampin's effect on the metabolism of antiretroviral drugs resulting in severely reduced levels of protease inhibitors (PIs), significantly reduced levels of the nonnucleoside reverse transcriptase inhibitor (NNRTI) nevirapine, and mild to moderate reductions in the level of efavirenz, another NNRTI.

The Centers for Disease Control and Prevention (CDC) has recently released guidelines on the management of drug interaction in the treatment of HIV-associated TB. ${ }^{35}$ In resource-limited settings, nevirapine-based regimens are used in most national ARV treatment programs but coadministration with rifampin is not recommended. Efavirenz-based ARV regimens are recommended in this scenario. However, it is unclear if standard dosing of efavirenz is adequate to compensate for any increases in efavirenz metabolism due to the concomitant use of rifampin. ${ }^{36}$ Matters become more complicated in the treatment of HIV-associated TB in pregnant women, a setting in which efavirenz is not recommended, and children, whose first-line ARV regimens often include PIs.

A few projects have assessed coadministration of these antituberculous and ARV agents with excellent preliminary results. ${ }^{34,37} \mathrm{~A}$ recent retrospective study in Thailand comparing HIV/TB coinfected individuals receiving rifampin-based TB therapy who started ARV therapy with either efavirenz or nevirapine at standard doses encouragingly showed no difference in virologic and CD4 outcomes between the two ARV treatment groups, though this study may have been underpowered. ${ }^{38}$ More pharmacokinetic and larger prospective and randomized clinical studies using various interventions and monitoring tools are urgently needed in patients receiving concomitant antituberculous and antiretroviral drugs to determine the exact risks and benefits of the various guidelines currently in use. The feasibility and effectiveness of rifabutin, which is less prone to drug interaction, in resource limited settings should also be urgently evaluated.

Paradoxical worsening of TB in HIV-negative patients after initiation of effective treatment for active TB is a long recognized clinical entity; in HIV-associated $\mathrm{TB}$ the rates of paradoxical worsening in patients on TB treatment who then start ARV therapy are much higher ${ }^{39}$ likely due to ARV therapy's beneficial effect on the immune system, and have been categorized as one of the many types of immune reconstitution inflammatory syndromes (IRIS) that patients with HIV are prone to developing after initiation of ARVs. ${ }^{40}$ The incidence of TB-IRIS in HIV/TB coinfected individuals receiving therapy for both diseases varies from study to study but is likely in the range of 20 to $30 \%$ of patients. ${ }^{41}$ TB-IRIS poses significant challenges to providers because there is no single diagnostic test that can distinguish TB-IRIS from treatment failure (an especially difficult problem in resource-limited settings where culture and drug susceptibility testing is often unavailable to help distinguish the two), no agreed upon case definition of IRIS, and no 
prospective or randomized trial data on how to best treat and manage TB-IRIS. More research in these areas is urgently needed.

Infection control measures for inpatient and outpatient settings need to be put into place whenever and wherever HIV-associated tuberculosis is being managed but perhaps most importantly in resource-limited settings, ${ }^{42}$ where the association between HIV and MDRand XDR-TB secondary to hospital-based transmission and spread has highlighted the need for better preventive practices. ${ }^{8}$ Improved infection control measures and increased program capacity in such settings are necessary to avoid further overwhelming the public health system. ${ }^{43}$ To be successful, however, not only will increased resources be necessary, but, in addition, TB and HIV treatment programs will need to collaborate in this effort. $^{44}$

\section{LATENT TUBERCULOSIS INFECTION}

\section{Diagnostic Challenges}

Despite its shortcomings, which include lower sensitivity in HIV-infected patients ${ }^{45,46}$ and cross-reactivity with components of the BCG vaccine, the TST is the accepted standard for diagnosis of LTBI. HIV-infected individuals with LTBI, as defined by lack of symptoms or signs consistent with active TB and with a positive TST, have a higher risk for reactivation than $\mathrm{HIV}$-uninfected individuals with LTBI. ${ }^{3-5,47}$ In many resource-limited settings, however, tuberculin skin testing is rarely available for a variety of reasons, including lack of access to clean needles and syringes for planting PPD and unavailability of properly refrigerated storage facilities for tuberculin.

In recent years, advances in LTBI diagnostics have been spurred by the elucidation of the Mycobacterium tuberculosis genome and the characterization of TBspecific antigens. ${ }^{48,49}$ These TB-specific antigens have led to the development of diagnostic tests for LTBI based on interferon-gamma production by T cells stimulated by these antigens, ${ }^{50}$ and have been dubbed interferon-gamma release assays (IGRAs). Three IGRAs are now commercially available: the QuantiFERON-TB Gold and the QuantiFERON-TB Gold In-Tube (Cellestis Inc., Valencia, CA), and the T-SPOT.TB (Oxford Immunotec Limited, Abingdon, Oxfordshire, UK) . The QuantiFERON is approved for use in the United States, with approval for the T-SPOT.TB expected to be imminent at the time of this writing. In this issue of Seminars, Drs. Pai and O'Brien (article 9) review these new diagnostics for LTBI. In brief, IGRAs have been shown, in immunocompetent adult subjects, to have excellent specificity for TB infection and good to excellent sensitivity for LTBI. The CDC has issued guidelines on the use of the QuantiFERON but cautions that it has not been extensively studied in children and immunocompromised individuals and that it may have a different sensitivity in these populations. ${ }^{51}$

Studies examining the performance of these new T cell-based IGRAs in immunocompromised and HIVinfected individuals have had mixed results. Some studies have shown the enzyme-linked immunosorbent assay (ELISA)-based tests to have an increased incidence of indeterminate results ${ }^{52-54}$ in these populations. Other studies have shown the evaluability and performance of the enzyme-linked immunospot (ELISPOT) assay-based tests to not be affected or to be affected to a lesser degree by the absolute CD4 $\mathrm{T}$ cell count, ${ }^{55-57}$ but a more recent study has challenged these findings. ${ }^{58}$ Larger studies examining the sensitivity, performance, and predictive value of IGRAs in patients with HIV are needed to allow providers to make the most informed decisions about who should receive treatment for LTBI.

\section{Treatment Challenges}

Adherence to and completion of treatment for LTBI are key to maximizing its effectiveness. Low perceived benefits of treatment increases the risk of noncompletion, ${ }^{59}$ but shorter regimens have been shown to improve completion rates. ${ }^{60}$ In this issue of Seminars, Dr. Sterling (article 6) reviews new strategies for the treatment of LTBI.

Randomized trials in resource-limited settings have shown that the use of isoniazid (INH) for treatment of LTBI in HIV-infected patients can reduce the incidence of $\mathrm{TB}^{61,62}$ and is cost-effective, ${ }^{63}$ but that benefit may wane over time, ${ }^{64}$ probably because of the high likelihood of reinfection in high TB burden settings. ${ }^{65}$ Another challenge to the successful treatment of LTBI in people infected with HIV in these settings includes a hesitancy on the part of many National TB Control Programs in implementing LTBI treatment programs using INH for fear that patients started on this regimen may, in fact, have undiagnosed active $\mathrm{TB}$, especially subclinical, smear-negative, or extrapulmonary $\mathrm{TB}$, and thus would inadvertently receive monotherapy for active TB disease resulting in emergence of drug resistant TB. Systematic and standardized screening for active $\mathrm{TB}$ in high $\mathrm{TB}$ burden and resource-limited settings leads to increased case detection ${ }^{17,66}$ but it is unclear how sensitive these strategies are and whether they can confidently be used to rule out all active TB disease prior to treatment for LTBI. Developing diagnostic tools and screening algorithms that can accurately diagnose LTBI in individuals with HIV while excluding active TB are areas needing further investigation.

\section{CONCLUSION}

HIV-related TB is a major global health threat. All currently available diagnostic methodologies in use are suboptimal in individuals with concomitant HIV 
infection. Concerted efforts are needed for the development of new rapid and accurate diagnostic tools for active TB disease and LTBI that perform well in patients with HIV disease.

In resource-limited settings where the greatest burden of HIV-associated TB exists, many of the tests described here, with the exceptions of the TST and sputum smear, are prohibitively expensive and/or require sophisticated laboratory infrastructure. Thus new diagnostic tools for TB and LTBI are needed that are practical, inexpensive, and feasible to implement in resource-limited settings.

Many unanswered questions and challenges also remain in the management and treatment of HIVrelated $\mathrm{TB}$, including the optimal timing of initiation of ARV therapy, the safest and least interaction-prone combinations of antituberculous and ARV drugs, and accurate and effective methods for diagnosis and management of TB-IRIS. Further clinical research, particularly prospective and randomized trials, is needed to answer the open questions in this area.

\section{REFERENCES}

1. World Health Organization. Global Tuberculosis Control: Surveillance, Planning, Financing. WHO Report. Geneva, Switzerland: WHO; 2008

2. UNAIDS. AIDS Epidemic Update: December 2007. Geneva, Switzerland: UNAIDS

3. Selwyn PA, Hartel D, Lewis VA, et al. A prospective study of the risk of tuberculosis among intravenous drug users with human immunodeficiency virus infection. [see comment] N Engl J Med 1989;320:545-550

4. Daley CL, Small PM, Schecter GF, et al. An outbreak of tuberculosis with accelerated progression among persons infected with the human immunodeficiency virus: an analysis using restriction-fragment-length polymorphisms. N Engl J Med 1992;326:231-235

5. Antonucci G, Girardi E, Raviglione MC, Ippolito G. Risk factors for tuberculosis in HIV-infected persons: a prospective cohort study. The Gruppo Italiano di Studio Tubercolosi e AIDS (GISTA). JAMA 1995;274:143-148

6. McIlleron H, Meintjes G, Burman WJ, Maartens G. Complications of antiretroviral therapy in patients with tuberculosis: drug interactions, toxicity, and immune reconstitution inflammatory syndrome. J Infect Dis 2007; 196(Suppl 1):S63-S75

7. World Health Organization. Anti-tuberculosis Drug Resistance in the World: Fourth Global Report. Geneva, Switzerland: WHO; 2008

8. Gandhi NR, Moll A, Sturm AW, et al. Extensively drugresistant tuberculosis as a cause of death in patients coinfected with tuberculosis and HIV in a rural area of South Africa. [see comment] Lancet 2006;368:1575-1580

9. Siddiqi K, Lambert ML, Walley J. Clinical diagnosis of smearnegative pulmonary tuberculosis in low-income countries: the current evidence. Lancet Infect Dis 2003;3:288-296

10. Colebunders RL, Ryder RW, Nzilambi N, et al. HIV infection in patients with tuberculosis in Kinshasa, Zaire. Am Rev Respir Dis 1989;139:1082-1085
11. Geng E, Kreiswirth B, Burzynski J, Schluger NW. Clinical and radiographic correlates of primary and reactivation tuberculosis: a molecular epidemiology study. JAMA 2005; 293:2740-2745

12. Harries $\mathrm{AD}$, Maher D, Nunn P. An approach to the problems of diagnosing and treating adult smear-negative pulmonary tuberculosis in high-HIV-prevalence settings in sub-Saharan Africa. Bull World Health Organ 1998;76:651662

13. De Cock KM, Soro B, Coulibaly IM, Lucas SB. Tuberculosis and HIV infection in sub-Saharan Africa. JAMA 1992;268: 1581-1587

14. Small PM, Schecter GF, Goodman PC, Sande MA, Chaisson RE, Hopewell PC. Treatment of tuberculosis in patients with advanced human immunodeficiency virus infection. N Engl J Med 1991;324:289-294

15. Hargreaves NJ, Kadzakumanja O, Whitty CJ, Salaniponi FM, Harries AD, Squire SB. 'Smear-negative' pulmonary tuberculosis in a DOTS programme: poor outcomes in an area of high HIV seroprevalence. Int J Tuberc Lung Dis 2001;5:847-854

16. Harries AD, Hargreaves NJ, Gausi F, Kwanjana JH, Salaniponi FM. High early death rate in tuberculosis patients in Malawi. Int J Tuberc Lung Dis 2001;5:10001005

17. Mtei L, Matee M, Herfort $\mathrm{O}$, et al. High rates of clinical and subclinical tuberculosis among HIV-infected ambulatory subjects in Tanzania. [see comment] Clin Infect Dis 2005; 40:1500-1507

18. Day JH, Charalambous S, Fielding KL, Hayes RJ, Churchyard GJ, Grant AD. Screening for tuberculosis prior to isoniazid preventive therapy among HIV-infected gold miners in South Africa. Int J Tuberc Lung Dis 2006;10: 523-529

19. Mohammed A, Ehrlich R, Wood R, Cilliers F, Maartens G. Screening for tuberculosis in adults with advanced HIV infection prior to preventive therapy. Int J Tuberc Lung Dis 2004;8:792-795

20. Cowie RL, Escreet BC. The diagnosis of pulmonary tuberculosis. S Afr Med J 1980;57:75-77

21. Singh KK, Dong Y, Hinds L, et al. Combined use of serum and urinary antibody for diagnosis of tuberculosis. J Infect Dis 2003;188:371-377

22. Singh KK, Zhang X, Patibandla AS, Chien PJr, ,Laal S. Antigens of Mycobacterium tuberculosis expressed during preclinical tuberculosis: serological immunodominance of proteins with repetitive amino acid sequences. Infect Immun 2001;69:4185-4191

23. Perkins MD, Conde MB, Martins M, Kritski AL. Serologic diagnosis of tuberculosis using a simple commercial multiantigen assay. Chest 2003;123:107-112

24. Breen RA, Hardy GA, Perrin FM, et al. Rapid diagnosis of smear-negative tuberculosis using immunology and microbiology with induced sputum in HIV-infected and uninfected individuals. PLoS One 2007;2:e1335

25. Ling DI, Flores LL, Riley LW, Pai M. Commercial nucleicacid amplification tests for diagnosis of pulmonary tuberculosis in respiratory specimens: meta-analysis and meta-regression. PLoS One 2008;3:e1536

26. Kibiki GS, Mulder B, van der Ven AJ, et al. Laboratory diagnosis of pulmonary tuberculosis in TB and HIV endemic settings and the contribution of real time PCR for M. tuberculosis in bronchoalveolar lavage fluid. Trop Med Int Health 2007;12:1210-1217 
27. Onyebujoh PC, Ribeiro I, Whalen CC. Treatment options for $\mathrm{HIV}$-associated tuberculosis. J Infect Dis 2007;196 (Suppl 1):S35-S45

28. Spigelman MK. New tuberculosis therapeutics: a growing pipeline. J Infect Dis 2007;196(Suppl 1):S28-S34

29. World Health Organization. Treatment of Tuberculosis: Guidelines for National Programmes 3rd ed. (English). WHO/CDS/TB/2003.313. Geneva, Switzerland: WHO; 2003

30. Blumberg HM, Burman WJ, Chaisson RE, et al. American Thoracic Society/Centers for Disease Control and Prevention/Infectious Diseases Society of America: treatment of tuberculosis. [see comment] Am J Respir Crit Care Med 2003; 167:603-662

31. World Health Organization. TB/HIV: A Clinical Manual. 2nd ed. Geneva, Switzerland: WHO; 2004

32. World Health Organization. Scaling up antiretroviral therapy in resource-limited settings: guidelines for a public health approach: executive summary. April 2002. IAPAC Mon 2002;8:168-175

33. Dean GL, Edwards SG, Ives NJ, et al. Treatment of tuberculosis in HIV-infected persons in the era of highly active antiretroviral therapy. [see comment] AIDS 2002;16: 75-83

34. Dheda K, Lampe FC, Johnson MA, Lipman MC. Outcome of $\mathrm{HIV}$-associated tuberculosis in the era of highly active antiretroviral therapy. [see comment] J Infect Dis 2004;190: 1670-1676

35. Centers for Disease Control and Prevention. Managing Drug Interactions in the Treatment of HIV-Related Tuberculosis [online]. 2007. Available at: http://www.cdc.gov/tb/TB_HIV_ Drugs/default.htm. Accessed May 31, 2008

36. Lopez-Cortes LF, Ruiz-Valderas R, Viciana P, et al. Pharmacokinetic interactions between efavirenz and rifampicin in HIV-infected patients with tuberculosis. Clin Pharmacokinet 2002;41:681-690

37. Jack C, Lalloo U, Abdool-Karim Q, et al. A pilot study of once-daily antiretroviral therapy integrated with tuberculosis directly observed therapy in a resource-limited setting. J Acquir Immune Defic Syndr 2004;36:929-934

38. Manosuthi W, Mankatitham W, Lueangniyomkul A, Chimsuntorn S, Sungkanuparph S. Standard-dose efavirenz vs. standard-dose nevirapine in antiretroviral regimens among HIV-1 and tuberculosis co-infected patients who received rifampicin. HIV Med 2008;9:294-299

39. Narita M, Ashkin D, Hollender ES, Pitchenik AE. Paradoxical worsening of tuberculosis following antiretroviral therapy in patients with AIDS. Am J Respir Crit Care Med 1998;158:157-161

40. Lawn SD, Bekker LG, Miller RF. Immune reconstitution disease associated with mycobacterial infections in HIVinfected individuals receiving antiretrovirals. [see comment] Lancet Infect Dis 2005;5:361-373

41. Burman W, Weis S, Vernon A, et al. Frequency, severity and duration of immune reconstitution events in HIV-related tuberculosis. Int J Tuberc Lung Dis 2007;11:1282-1289

42. Bock NN, Jensen PA, Miller B, Nardell E. Tuberculosis infection control in resource-limited settings in the era of expanding HIV care and treatment. J Infect Dis 2007; 196(Suppl 1):S108-S113

43. Wells CD, Cegielski JP, Nelson LJ, et al. HIV infection and multidrug-resistant tuberculosis: the perfect storm. J Infect Dis 2007;196(Suppl 1):S86-S107
44. Andrews JR, Shah NS, Gandhi N, Moll T, Friedland G. Tugela Ferry Care and Research C: multidrug-resistant and extensively drug-resistant tuberculosis: implications for the HIV epidemic and antiretroviral therapy rollout in South Africa. J Infect Dis 2007;196(Suppl 3):S482-S490

45. Screening for tuberculosis and tuberculous infection in highrisk populations. Recommendations of the Advisory Committee for Elimination of Tuberculosis. MMWR Recomm Rep 1990;39(RR-8):1-7

46. Markowitz N, Hansen NI, Wilcosky TC, et al. Tuberculin and anergy testing in HIV-seropositive and HIV-seronegative persons. Pulmonary Complications of HIV Infection Study Group. [see comment] Ann Intern Med 1993;119: $\quad$ 185-193

47. Guelar A, Gatell JM, Verdejo J, et al. A prospective study of the risk of tuberculosis among HIV-infected patients. AIDS 1993;7:1345-1349

48. Louise R, Skjøt R, Agger E, Anderson P. Antigen discovery and tuberculosis vaccine development in the post-genomic era. Scand J Infect Dis 2001;33:643-647

49. Gey Van Pittius NC, Gamieldien J, Hide W, Brown GD, Siezen RJ, Beyers AD. The ESAT-6 gene cluster of Mycobacterium tuberculosis and other high $\mathrm{G}+\mathrm{C}$ gram-positive bacteria. Genome Biol 2001;2(10):RESEARCH0044

50. Mori T, Sakatani M, Yamagishi F, et al. Specific detection of tuberculosis infection: an interferon-gamma-based assay using new antigens. [see comment] Am J Respir Crit Care Med 2004;170:59-64

51. Mazurek GH, Jereb J, Lobue P, et al. Guidelines for using the QuantiFERON-TB Gold test for detecting Mycobacterium tuberculosis infection, United States. [erratum appears in MMWR Morb Mortal Wkly Rep. 2005 Dec 23;54(50): 1288]. MMWR Recomm Rep 2005;54(RR-15):49-55

52. Tsiouris SJ, Coetzee D, Toro PL, Austin J, Stein Z, El-Sadr W. Sensitivity analysis and potential uses of a novel gamma interferon release assay for diagnosis of tuberculosis. J Clin Microbiol 2006;44:2844-2850

53. Kobashi Y, Mouri K, Obase Y, Fukuda M, Miyashita N, Oka M. Clinical evaluation of QuantiFERON TB-2G test for immunocompromised patients. Eur Respir J 2007;30:945950

54. Luetkemeyer AF, Charlebois ED, Flores LL, et al. Comparison of an interferon-gamma release assay with tuberculin skin testing in HIV-infected individuals. Am J Respir Crit Care Med 2007;175:737-742

55. Dheda K, Lalvani A, Miller RF, et al. Performance of a Tcell-based diagnostic test for tuberculosis infection in HIVinfected individuals is independent of CD4 cell count. [see comment] AIDS 2005;19:2038-2041

56. Hoffmann M, Reichmuth M, Fantelli K, et al. Conventional tuberculin skin testing versus $\mathrm{T}$-cell-based assays in the diagnosis of latent tuberculosis infection in HIV-positive patients. [comment] AIDS 2007;21:390-392

57. Rangaka MX, Diwakar L, Seldon R, et al. Clinical, immunological, and epidemiological importance of antituberculosis $\mathrm{T}$ cell responses in HIV-infected Africans. Clin Infect Dis 2007;44:1639-1646

58. Karam F, Mbow F, Fletcher H, et al. Sensitivity of IFNgamma release assay to detect latent tuberculosis infection is retained in HIV-infected patients but dependent on HIV/ AIDS progression. PLoS One 2008;3:e1441

59. Shieh FK, Snyder G, Horsburgh CR, Bernardo J, Murphy C, Saukkonen JJ. Predicting non-completion of treatment for 
latent tuberculous infection: a prospective survey. Am J Respir Crit Care Med 2006;174:717-721

60. Lardizabal A, Passannante M, Kojakali F, Hayden C, Reichman LB. Enhancement of treatment completion for latent tuberculosis infection with 4 months of rifampin. [see comment] Chest 2006;130:1712-1717

61. Hawken MP, Muhindi DW. Tuberculosis preventive therapy in HIV-infected persons: feasibility issues in developing countries. Int J Tuberc Lung Dis 1999;3:646-650

62. Wilkinson D. Drugs for preventing tuberculosis in HIV infected persons. Cochrane Database Syst Rev 2000; (4):CD000171 Update in: Cochrane Database Syst Rev 2004;(1):CD000171; PMID: 14973947

63. Bell JC, Rose DN, Sacks HS. Tuberculosis preventive therapy for HIV-infected people in sub-Saharan Africa is cost-effective. [see comment] AIDS 1999;13:15491556

64. Johnson JL, Okwera A, Hom DL, et al. Duration of efficacy of treatment of latent tuberculosis infection in HIV-infected adults. AIDS 2001;15:2137-2147

65. Sonnenberg P, Murray J, Glynn JR, Shearer S, Kambashi B, Godfrey-Faussett P. HIV-1 and recurrence, relapse, and reinfection of tuberculosis after cure: a cohort study in South African mineworkers. [see comment][erratum appears in Lancet 2002 Jun 15;359(9323):2120] Lancet 2001;358:16871693

66. Gasana M, Vandebriel G, Kabanda G, et al. Integrating tuberculosis and HIV care in rural Rwanda. Int J Tuberc Lung Dis 2008;12(3 Suppl 1):S39-S43 